Revista de Dialectología y Tradiciones Populares, vol. LXXIII, n. ${ }^{\circ}$ 2, pp. 287-294, julio-diciembre 2018,

ISSN: 0034-7981, eISSN: 1988-8457, https://doi.org/10.3989/rdtp.2018.02.001

\title{
EDITORIAL
}

\section{Mudar de nombre}

\section{Changing name}

\author{
Pedro Tomé1 \\ Instituto de Lengua, Literatura y Antropología (ILLA)-CSIC \\ Francisco Ferrándiz ${ }^{2}$ \\ Instituto de Lengua, Literatura y Antropología (ILLA)-CSIC
}

\section{RESUMEN}

En el presente texto se explica el próximo cambio de nombre de la Revista de Dialectología y Tradiciones Populares por el de Disparidades. Revista de Antropologia, con el objetivo de adaptarse a la nueva política editorial y, fundamentalmente, a las transformaciones de la disciplina antropológica en las últimas décadas. Aunque el título original atesora un indudable capital científico, el Consejo de Redacción de la revista ha considerado que ha llegado el momento de sustituirlo por uno más actualizado.

Palabras clave: Revistas académicas; Editoriales académicas; Antropología social y cultural.

\section{SUMMARY}

This text argues for the upcoming change of the name of the journal from Revista de Dialectologia y Tradiciones Populares to Disparidades. Revista de Antropología, in order to adapt it to the current and future editorial line of the journal as well as to the transformation of the anthropological discipline in the last few decades. Although the original title possesses an unquestionable academic capital, we believe it is the right moment to update it.

Keywords: Academic Journals; Academic Presses; Sociocutural Anthropology.

En 1944, en el seno del Instituto Antonio de Nebrija de Filología Clásica, sería fundada la Revista de Tradiciones Populares. Un año más tarde, 1945, mudaría su nombre por el que aún mantiene hoy día: Revista de Dialectología y Tradiciones Po-

\footnotetext{
${ }^{1}$ Correo electrónico: pedro.tome@cchs.csic.es. ORCID iD: <https://orcid.org/0000-0001-5380-5994>.

${ }^{2}$ Correo electrónico: paco.ferrandiz@cchs.csic.es. ORCID iD: <http://orcid.org/0000-0002-4095-6344>.
} 
pulares. Desde un primer momento, siguiendo la concepción dominante en la época, la revista se orientaría hacia una comprensión filológica del folklore y, como ha mostrado Luis Calvo (2002), se caracterizaría por dar a conocer investigaciones que expresaban un concepto socialmente conservador de la tradición y la cultura popular. Vinculada hasta comienzos de la década de los sesenta al Centro de Estudios de Etnología Peninsular del CSIC —y, posteriormente, al Departamento de Dialectología y Tradiciones Populares del Instituto Miguel de Cervantes de Filología Hispánica-, la revista fue incorporando progresivamente estudios de carácter antropológico, si bien sin abandonar su orientación teórico-ideológica como han señalado tanto Concepción Casado (1988), quien fuera directora de la revista mediada la década de los 90, como Carmen Ortiz (1994), miembro de su Consejo de Redacción desde 1984 y directora de la misma ya en el siglo XXI.

La llegada de Julio Caro Baroja a la dirección de la revista en 1978 supuso una apertura metodológica y disciplinar a las nuevas tendencias de la antropología cultural. Con el explícito objetivo de hacerla más acorde a los tiempos que en ese momento se vivían, particularmente desde 1984, Caro Baroja, con el concurso del Consejo de Redacción de la revista, llevaría a cabo una transformación radical de sus contenidos incorporando nuevas metodologías y temas propios de la antropología social y cultural de aquel momento. No obstante una renovación tan significativa, las secciones que la conformaban apenas sufrieron modificaciones y los componentes filológico y folklórico de la revista se mantuvieron inalterados conviviendo con las innovaciones. La revista siempre ha estado en movimiento, y nuevos cambios fueron acumulándose con el tiempo. Así, como ejemplo destacable, a comienzos de la década de los 90 del pasado siglo XX comenzaron a aparecer volúmenes temáticos dedicados de forma monográfica a cuestiones específicas y, sobre todo, con una orientación más clara hacia la antropología social contemporánea, que seguía conviviendo con los otros componentes históricos de la revista.

No tratamos, en todo caso, de escribir en estas líneas una historia de la Revista de Dialectología y Tradiciones Populares, por otra parte ya sistematizada para sus primeras épocas por los autores citados (Casado 1988; Ortiz 1994; Calvo 2002), así como parcialmente por otros colegas. Más bien, nuestro interés es señalar que, más allá de épocas de inmovilismo y otras de grandes cambios, la Revista de Dialectología y Tradiciones Populares ha mantenido un cierto dinamismo desde su creación hace ya más de setenta años hasta nuestros días. Un dinamismo que la ha llevado desde un arranque que priorizó la filología propugnada por su fundador, Vicente García Diego, a incluir en su portada, desde 2006 bajo la dirección de Antonio Cea, que es una revista dedicada a Antropología, Etnografía y Folklore y, por último, a una nueva renovación acontecida en 2015 en la que se marcaba una orientación exclusivamente antropológica.

En el "Editorial" con que arrancaba el volumen 70 de la Revista de Dialectología y Tradiciones Populares, firmado por Tomé y Ferrándiz, se explicaba una nueva modulación en la línea editorial aseverando que

Desde ese compromiso con lo público, el nuevo proyecto editorial 2015-2018, que se abre con este número y cuyos cambios se irán completando en los sucesivos, pretende continuar la senda de modernización temática y conceptual de la revista, adecuándola paulatinamente a las tendencias y preocupaciones más recientes de la antropología social y cul- 
tural del siglo XXI. Sobre el fermento de su base histórica —especialmente la línea editorial de la última década-, se incorporarán de manera prioritaria textos que contribuyan a dibujar la antropología del presente y del futuro (Tomé y Ferrándiz 2015: 8).

Como consecuencia de esos cambios, desde ese momento, como se indica en su web, la RDTP

es una publicación científica que valora especialmente las contribuciones innovadoras en la investigación antropológica, entendiéndola en un sentido teórico y metodológico amplio y plural. Publica artículos originales que, además de contribuir al conocimiento antropológico, enriquezcan el debate crítico entre los investigadores, con especial atención a las aportaciones teóricas, metodológicas y a la etnografía de campo (RDTP 2018).

El nuevo equipo editorial que se formó en 2015, y que desde entonces dirige la revista, está conformado, tanto en su Consejo de Redacción como en el Asesor, exclusivamente por profesionales de la antropología del propio CSIC, así como de universidades de España (Universidad Complutense de Madrid, Universidad Autónoma de Madrid, Universidad Nacional de Educación a Distancia, Universidad de Barcelona, Universidad Autónoma de Barcelona, Universidad del País Vasco, Universidad de Granada, Universidad de Sevilla, Universidad Rovira i Virgili de Tarragona, Universidad de Lleida), Estados Unidos (University of Michigan, Brown University, University of North Texas), Reino Unido (University of Oxford, Nottingham Trent University, The University of Manchester), Portugal (ISCTE-Instituto Universitario Lisboa), Brasil (Universidade Federal de Santa Catarina), México (Centro de Investigaciones y Estudios Superiores en Antropología Social - CIESAS-) y Argentina (Universidad de Buenos Aires).

Fruto de la nueva orientación, además de portadas alusivas a los contenidos de cada volumen, es la aparición de nuevas secciones. Así junto a las ya consolidadas de "Artículos de investigación", "Fuentes y Testimonios" y "Notas de Libros", otras han sido incorporadas a las revistas, ya sea en una modalidad fija o esporádica. Consideramos de especial importancia la consolidación de las secciones "Cartografías" y "Temas Emergentes".

Entre 2015 y 2018 se habrán publicado cuatro "Cartografías", una cada año y, dada la dificultad de redactar este tipo de textos, están ya previstas las de los tres próximos años. Cada una de las ya publicadas ha llevado a cabo una suerte de estado de la cuestión sobre diferentes áreas temáticas de la antropología social y cultural que, además de valorar el conjunto de investigaciones sobre un tema concreto, trataba de imaginar y cartografiar nuevos escenarios de investigación en cada ámbito específico tratado. Así, el volumen 70-1 incluyó una "Genealogía de la Antropología Médica en España" (Martínez-Hernáez, Perdiguero y Comelles 2015) en la que, desde una perspectiva histórica, se analizan las diferentes etapas por las que han pasado dichos estudios. Además del recorrido histórico a través de diferentes fases, el artículo incluye un debate abierto sobre el futuro de la antropología médica en España centrándose en los retos que le plantean las nuevas identidades - y bioidentidades- que están surgiendo en los procesos de medicalización desarrollados en un contexto global. En el volumen 71-2 la cartografía se enfocó hacia los "Usos y significados contemporáneos de la comida desde la antropología de la alimentación en América Latina y España" (López, Mariano y Medina 2016). En la misma, agrupándose en tres ejes —pa- 
trimonio alimentario, entre localidad y globalización; carencias alimentarias; y simbolismos y significaciones alimentarias - los autores proponen una exhaustiva revisión de la antropología de la alimentación en España y en Latinoamérica desde una perspectiva histórica reciente. Un año después, José Antonio Langarita Adiego y Jordi Mas Grau planteaban un cartografía sobre "Antropología y diversidad sexual y de género en España. Hacia la construcción de una especialidad disciplinaria" (Langarita y Mas 2017). Más allá de una revisión bibliográfica sobre el recorrido, las transformaciones y los ámbitos de interés de los trabajos sobre diversidad sexo-genérica en la antropología española desde 1980 hasta 2017, muestran, según sus autores, la amplia variedad de temáticas que se abordan en esta especialidad disciplinaria en la que, además de los estudios sobre la homosexualidad, han surgido nuevas áreas de interés, tales como los estudios trans- e intersex que, aún con influencia internacional, también poseen un recorrido propio en España. Por último, en este mismo volumen se publica una cuarta cartografía centrada en esta ocasión en "La antropología de los contextos musulmanes desde España: inmigración, islamización e islamofobia" (Téllez y Ramírez 2018) En la misma las autoras, tratando de reconstruir la racionalidad de temas y de enfoques, así como de identificar regularidades, plantean un recorrido por las aportaciones de la antropología de los contextos musulmanes en el panorama académico del Estado español. Además de ofrecer una boja de ruta para orientarse por la enorme producción existente sobre la cuestión, las autoras proponen algunos debates para situar a la antropología en ámbitos que, trascendiendo lo académico, permitan su inserción en ámbitos de producción de discursos públicos crítica y políticamente construidos.

Durante estos cuatro años (2015-2018), la Revista de Dialectología y Tradiciones Populares también ha publicado otros tantos "Temas Emergentes". Esta sección de clara orientación pública parte del interés del Consejo de Redacción por abordar con la mayor agilidad posible temas de actualidad en los que la antropología social y cultural puede aportar un punto de vista para el debate académico, social y político. La sección está coordinada por uno o varios autores y se compone de una secuencia de textos cortos por parte de especialistas nacionales e internacionales sobre el tema concreto que se propone. El dossier inaugural de esta sección, denominado "Migración y refugio en el mediterráneo, más allá de las fronteras» estuvo coordinado por Liliana Suárez-Navaz (2015). En él, desde una perspectiva crítica, se analizan tanto las políticas europeas de asilo y la economía moral que subyace a ellas, como las retóricas del humanitarismo que mueven el movimiento asociativo y que en ocasiones priorizan los conceptos de crisis y urgencia - dejando fuera de foco la justicia social y los derechos humanos; y la cobertura mediática, que espectaculariza todo el proceso y a la postre arroja más sombras que luces sobre la tragedia, que está teniendo lugar en el Mediterráneo-. En "Colaboraciones experimentales: una modalidad etnográfica", Adolfo Estalella y Tomás Sánchez Criado (2016) y los autores invitados al dossier plantean una discusión sobre cómo reimagninar y reaprender la práctica etnográfica en el mundo contemporáneo, en el que el tradicional encuentro etnográfico ya no es un paradigma de relación viable sobre el terreno. Plantean la necesidad de renovar el vocabulario descriptivo y el registro conceptual del trabajo de campo etnográfico y repensar al tiempo los procesos complejos de producción de conocimiento derivados de él. En "La emigración juvenil, ¿aventura o exilio?» Carles Feixa y 
Clara Rubio (2017) bosquejan una mirada antropológica sobre el éxodo juvenil en nuestro país motivado por la falta de perspectivas laborales, la precarización del empleo y la ausencia de un horizonte vital viable. La más reciente, coordinada por Marta Pérez y Ainhoa Montoya (2018), ha puesto el foco en otro tema de una importante dimensión social y consecuencias dramáticas para las generaciones más jóvenes de profesores e investigadores: las "precariedades en la Academia". Las autoras enmarcan el debate en la importancia de conectar las experiencias personales con un proceso global de neoliberalización de la Academia. Este último tema emergente presenta artículos en castellano e inglés, e incluye por primera vez una introducción bilingüe en la versión online de la revista. Al igual que en las "Cartografías", ya hay propuestas aprobadas por el Consejo de Redacción para las próximas entregas de esta nueva sección. Hay que mencionar que, habida cuenta sus características, y si bien la revista se dirige, como se recoge en su web, "a un público de carácter internacional especializado" en la antropología, la sección "Temas Emergentes" ha tenido un cierto impacto más allá de lo estrictamente académico, tanto a través de las redes sociales como en los medios de comunicación más clásicos. Así, la sección coordinada por Liliana Suárez en 2015 dio lugar a un artículo en el periódico digital Público —en su sección "Universidad del Barrio" se entrevistaba a la coordinadora del dossier y se enlazaba a la página web de la revista ${ }^{3}$, lo que supuso un gran incremento de visitas a la misma- El Consejo de Redacción de la revista valoró positivamente esta iniciativa, y tratará de que el número máximo de temas emergentes tengan una difusión pública similar. También han tenido incidencia en las redes sociales algunos de los artículos que caracterizan la orientación de la revista, como los dedicados a los desplazados por conflictos o los migrantes, los nuevos modelos familiares, los nuevos modelos patrimonializadores, el urbanismo, las prácticas religiosas, las reflexiones sobre el cuerpo o, por supuesto, varias de las discusiones teóricas aparecidas en los distintos números. No ha sido intención expresa de la revista lograr tener grandes impactos en las redes sociales o que estos sustituyan a las cuestionables métricas académicas. Pero hemos constatado algunas de las virtudes (no exentas de riesgos) que de ellos se pueden derivar, por lo que sería absurdo renunciar a estos medios — nunca finesque facilitan la comunicación y participación de numerosos potenciales lectores.

Otras dos secciones también han aparecido en estos últimos años. Nos referimos a las que llevan por nombre "En pocas palabras" y, más recientemente, "En tierra de nadie». La primera de ellas está pensada para propiciar breves reflexiones, debates, críticas, balances de congresos, noticias, etc. Ejemplo de ello es la noticia sobre el II Seminario Red de Antropología Ambiental que con el título "Etnografías de las relaciones humano-animales" escribieron Cruzada, Campanera y Escalera (2017). Por último, en la sección "En tierra de nadie" (In the Wild", en su nombre en inglés) se incluyen textos que por sus características especiales no tienen cabida en las secciones regulares pero que se consideran de interés para las y los lectores de la revista. Ejemplo de ello puede ser el artículo "Retóricas del momento: (per)versiones (mis)antropológicas" (Ruiz y Moncusí 2018), que recoge la conferencia de clausura del XIV Congreso de Antropolo-

\footnotetext{
${ }^{3}$ Ver <https://blogs.publico.es/universidad-del-barrio/2016/04/24/migracion-y-refugio-en-elmediterraneo-los-agujeros-negros-de-la-modernidad-abren-la-serie-de-temas-emergentes-en-la-revistadel-csic/>. Fecha de acceso: 20 sep. 2018.
} 
gía de la FAAEE celebrado en Valencia en 2017. En ella, un supuesto antropólogo de una universidad africana - Vicente Artur Belda-, paella y Nino Bravo mediante, reflexiona sobre las discusiones teóricas en el trabajo de campo o el compromiso que adquiere el etnógrafo con lo que considera que son sus causas emancipadoras. La originalidad de esta ficción etnográfica, leída por un actor en la clausura de un congreso nacional, hizo que nos pusiéramos en contacto con sus autores reales - desconocidos para los asistentes durante el acto de su lectura- para sugerir su publicación en nuestra revista. Los autores aceptaron la invitación y le añadieron el aparato crítico, sin tocar la conferencia tal y como se leyó públicamente. Con este artículo la revista se abre también a la inclusión de textos más experimentales y heterodoxos.

En suma, como puede apreciarse en las líneas precedentes, la renovación de la línea editorial supone que, actualmente, la revista publique mayoritariamente artículos que tienen que ver con las tendencias y preocupaciones más recientes de la antropología social y cultural del siglo XXI, lo que implica una discordancia fácilmente constatable con el nombre de la propia revista. La disonancia surgida de este anacronismo entre el nombre de la revista y sus contenidos genera problemas de tres tipos.

Por una parte, el título actual hace que la revista pueda ser percibida como un híbrido y tenga dificultades para ubicarse con nitidez en el conjunto de revistas de antropología social contemporáneo. Desde el punto de vista de su ubicación en los índices de impacto, de importancia muy significativa en el entorno académico presente, el nombre actual también puede resultar engañoso y no favorece su catalogación en los índices más específicamente antropológicos. En segundo lugar, la revista sigue recibiendo numerosos artículos propios de la dialectología o de las tradiciones populares que deben ser rechazados por su inadecuación temática con el nuevo proyecto editorial y la evolución de la propia disciplina (a pesar de su adecuación al título). Esto genera, además de confusiones y pérdidas de tiempo y energía tanto en los autores como en la propia revista, protestas por parte algunos autores que envían sus aportaciones a una revista que creen propia de su campo debido al nombre que ostenta en la cabecera. Finalmente, no son pocos los profesionales de la antropología, principalmente de otros países donde todavía no está suficientemente posicionada, que mantienen una cierta resistencia a publicar en una revista que se considera de dialectología, pues puede introducir un cierto ruido en su carrera.

Atendiendo a lo expuesto, tras varios meses de deliberación y decenas de propuestas de nombres alternativos, el Consejo de Redacción de la revista acordó solicitar al director de Editorial CSIC que, coincidiendo con el septuagésimo quinto aniversario de la creación de la misma, se autorizase el cambio de nombre de la Revista de Dialectología y Tradiciones Populares sustituyéndolo por el de Disparidades. Revista de Antropología. Con dicho acto se prosigue en la dirección que se indicaba en el citado "Editorial" (Tomé y Ferrándiz 2015) en la que se indica que la revista iría asumiendo paulatinamente cambios para "continuar la senda de modernización temática y conceptual de la revista" iniciada décadas atrás. La línea editorial fijada en ese momento asume como punto de partida que la antropología no es solo una fuente de conocimiento empírico y teórico basada en la experiencia directa e intensiva de los entornos de investigación estudiados, sino también un agente crítico y transformador de la percepción de la complejidad de los procesos sociales, económicos, culturales y ambientales. Por tal motivo, resulta imprescindible que preste atención, jun- 
to a los temas clásicos de la disciplina, a aquellos que tienen que ver con la diversidad cultural expresada de múltiples y complejas formas que pueden incluir semejanzas y desigualdades. Es decir, compaginar de manera imaginativa la versatilidad del repertorio metodológico de la disciplina, su constante labor teórica y analítica, y su intuición para detectar y acotar los entornos de investigación relevantes en cada contexto - lo que algunos autores denominan "imaginación etnográfica»—, no lleva a desconsiderar homogeneidades, identidades e igualdades, sino a descubrir las formas plurales y dispares en que estas pueden llegar a construirse. Además, al considerar la etnografía como un proceso emergente y denso en el que se establecen dinámicas de retroalimentación entre teoría y práctica, entre realidad y texto, entre diseños de investigación y situaciones cambiantes, entre escenarios de campo y aplicación de técnicas de investigación, entre la posición del investigador y la de los informantes, entre los investigadores y las audiencias de sus textos, etcétera, lo dispar y las disparidades se convierten en escenario idóneo para la reflexión y el avance antropológico. Igualmente, el nombre Disparidades. Revista de Antropología, además de fijar claramente y sin ambigüedad el área de conocimiento al que se dirige la revista, concuerda plenamente con estrategias de naming propias de la disciplina, al situarla en una línea que mantienen otras revistas ya clásicas y de gran proyección. Una estrategia que se acompaña, además, del abandono del papel como soporte fundamental y su sustitución por el formato electrónico de manera exclusiva. Es una forma más de reducir la factura ambiental y, aunque sea de modo simbólico, no contribuir al gasto de agua y energía más de lo estrictamente imprescindible. Este cambio, no obstante, no se verá reflejado en la numeración: el próximo número, ya con el nombre nuevo, será el 74 y no el uno o el cero.

No quisiéramos cerrar este editorial sin agradecer la continua ayuda de la Unidad de Apoyo a la Edición de Revistas del Centro de Ciencias Humanas y Sociales del CSIC y, sobre todo, el aliento y amparo que la Editorial CSIC, personalizada en su Director y en el Responsable del Servicio de Edición Electrónica, nos ha brindado en todo momento y sin el cual los cambios reseñados, y los que en el futuro próximo vendrán, no hubieran sido posibles.

\section{BIBLIOGRAFÍA CITADA}

Calvo, Luis. 2002. "La Revista de Dialectología y Tradiciones Populares y sus aportaciones a la antropología española (1944-1996)". Revista de Dialectología y Tradiciones Populares 57(1): 29-58. Disponible en: <http://rdtp.revistas.csic.es/index.php/rdtp/article/view/188/189>.

Casado, Concepción. 1988. "La Revista de Dialectología y Tradiciones Populares", en Jean-Pierre Piniès, Christiane Amiel y René Piniès (eds.), Au miroir des revues. Ethnologie de l'Europe du Sud: 103-107. Cahiers d'Ethnologie Méditerranéenne 1. Carcassonne: Garae/Hesiode.

Cruzada, Santiago, Mireia Campanera y Javier Escalera. 2017. "Etnografías de las relaciones humano-animales. II Seminario Red de Antropología Ambiental”. Revista de Dialectología y Tradiciones Populares 72(2): 527-532. doi: <https://doi.org/10.3989/rdtp.2017.02.010>.

Estalella, Adolfo y Tomás Sánchez Criado (coords.). 2016. "Colaboraciones experimentales: una modalidad etnográfica". Revista de Dialectología y Tradiciones Populares 71(1): 9-73. doi: <https:/ /doi.org/10.3989/rdtp.2016.v71.i1>.

Feixa, Carles y Clara Rubio (coords.). 2017. "La emigración juvenil, ¿aventura o exilio?». Revista de Dialectología y Tradiciones Populares 72(1): 9-66. doi: <https://doi.org/10.3989/rdtp.2017.v72.i1>. 
Langarita Adiego, José Antonio y Jordi Mas Grau. 2017. "Antropología y diversidad sexual y de género en España. Hacia la construcción de una especialidad disciplinaria". Revista de Dialectología $y$ Tradiciones Populares 72(2): 311-334. doi: <https://doi.org/10.3989/rdtp.2017.02.001>.

López, Julián, Lorenzo Mariano Juárez y F. Xavier Medina. 2016. “Usos y significados contemporáneos de la comida desde la antropología de la alimentación en América Latina y España". Revista de Dialectologia y Tradiciones Populares 71(2): 327-370. doi: <https://doi.org.10.3989/ rdtp.2016.02.001>.

Martínez-Hernáez, Ángel, Enrique Perdiguero y Josep María Comelles. 2015. "Genealogía de la Antropología Médica en España". Revista de Dialectología y Tradiciones Populares 70(1): 205-233. doi <https://doi.org/10.3989/rdtp.2015.01.010>.

Ortiz, Carmen. 1994. "Revista de Dialectología y Tradiciones Populares", en Carmen Ortiz y Luis Ángel Sánchez (eds.). Diccionario histórico de la antropología española: 581-584. Madrid: CSIC.

Pérez, Marta y Ainhoa Montoya (coords.). 2018. "Precariedades en la Academia". Revista de Dialectología y Tradiciones Populares 73(1): 9-61. doi: <https://doi.org/10.3989/rdtp.2018.v73.i1>.

RDTP [Revista de Dialectología y Tradiciones Populares]. 2018. <http://rdtp.revistas.csic.es/index.php/ rdtp>. Fecha de acceso: 20 sep. 2018.

Ruiz, Miquel A. y Albert Moncusí. 2018. "Retóricas del momento: (per)versiones (mis)antropológicas". Revista de Dialectología y Tradiciones Populares 73(1): 63-85. doi: <https://doi.org/10.3989/ rdtp.2018.01.002>.

Suárez-Navaz, Liliana (coord.). 2015. "Migración y refugio en el mediterráneo, más allá de las fronteras". Revista de Dialectologia y Tradiciones Populares 70(2): 265-336. doi: <https://doi.org/ 10.3989/rdtp.2015.v70.i2>.

Tomé, Pedro y Francisco Ferrándiz. 2015. «Editorial”. Revista de Dialectología y Tradiciones Populares 70(1): 7-10. Disponible en: <http://rdtp.revistas.csic.es/index.php/rdtp/article/view/463/468>.

Téllez, Virtudes y Ángeles Ramírez. 2018. "La antropología de los contextos musulmanes desde España: inmigración, islamización e islamofobia». Revista de Dialectología y Tradiciones Populares 73(2): 295-324. doi: <https://doi.org/10.3989/rdtp.2018.02.002>. 